\title{
Simple Three-Input Single-Output Current-Mode Universal Filter Using Single VDCC
}

\author{
Prungsak Uttaphut \\ Department of Electrical Technology, Faculty of Industrial Technology, Suan Sunandha Rajabhat University, Thailand
}

\begin{tabular}{l}
\hline \hline Article Info \\
\hline Article history: \\
Received Apr 1, 2018 \\
Revised Jun 4, 2018 \\
Accepted Jul 22, 2018 \\
\hline
\end{tabular}

\section{Keyword:}

Active filter

commercially available IC

Electronic control

Universal biquad

VDCC

\begin{abstract}
This paper presents a second-order current-mode filter with three-inputs and single-output current using single voltage differencing current conveyors (VDCC) along with one resistor and two grounded capacitors. The design of presented filter emphasizes on the use of a single active element without the multiple terminals VDCC which is convenient to implement the VDCC using commercially available IC for the practical test. Also, it can reduce the current tracking error at current output port and can reduce the number of transistors in the VDCC. The proposed filter can realize all the five generic filter responses, namely, band-pass (BP), band-reject (BR), low-pass (LP), high-pass (HP), and all-pass (AP) functions from the same configuration under various conditions in terms of three input current signals. Furthermore, the natural frequency and quality factor are electronically controlled. The output current node exhibits high impedance. Besides, the non-ideal case is also investigated. The simulation and experimental results using VDCC constructed from commercially available IC can validate the theoretical analyses.
\end{abstract}

Copyright $@ 2018$ Institute of Advanced Engineering and Science. All rights reserved.

\section{Corresponding Author:}

Prungsak Uttaphut,

Department of Electrical Technology, Faculty of Industrial Technology,

Suan Sunandha Rajabhat University,

1 U-Thong Nok Road, Dusit, Bangkok 10300, Thailand.

Email: prungsak.ssru@hotmail.com

\section{INTRODUCTION}

Analog filters are an important building blocks for analog signal processing that have a variety of applications in the fields of communication, sound system, instrumentation, control system, etc [1]. The filter which provides several filter responses in the same topology is well-known as the universal filter or multifunction filter. The multifunction filter with multiple inputs and single output is one of the universal filters which have attracted significant research attention [2]. In addition to these, a current-mode (CM) signal processing circuits in which some parameters can be controlled by external currents have low power consumption, greater linearity, a smaller number of components and larger dynamic range, wider bandwidth when compared to voltage-mode counterparts such as operational amplifiers [3]-[4].

The design of an electronic circuit in analog signal processing has gone in the use of the active building block [5]-[9]. Especially, the electronically tunable active building blocks have attracted significant research attention since analog circuits using electronically tunable active building block give more finetuning than adjusting the value of passive device. The voltage differencing current conveyors (VDCC) [10] is a recently reported versatile active building block used in the realization of analog signal processing circuits. A very significant advantage of using VDCCs in analog circuit design is that it is able electronic controllability.

Analog circuits using the VDCC as the active element have been found in the literature for examples, the first order allpass filter [11], the ladder filter [12], the passive element simulator [10], [13]- 
[16], the square and triangular wave generator [17], sinusoidal oscillator [18]-[21], etc. The VDCC based universal filters have been proposed in [22]-[25]. The three-input single-output (TISO) voltage-mode filter and single-input dual-output (SIDO) voltage-mode filters were presented in [22]. The TISO filter with five filter responses consists of single VDCC, one resistor and two capacitors. The SIDO filters with three filter responses (HP, BP and LP) consist of single VDCC, two resistors and two capacitors. The natural frequency and quality factor for both TISO and SIDO are electronically tuned. Moreover, the tune of quality factor for SIDO filter can be done without affecting the natural frequency. However, the output voltage node doesn't exhibit low impedance, so the voltage buffer is required for cascading. The single-input four-output voltagemode filter was proposed in [23]. It consists of single VDCC, two resistors and two capacitors. The natural frequency and quality factor are electronically tuned. Moreover, the tune of quality factor can be done without affecting the natural frequency. However, the output voltage nodes don't exhibit low impedance, so the voltage buffer is required for cascading. The current-mode reconnection-less reconfigurable filter was proposed in [24]. It consists of single VDCC, a single dual output current amplifier, a single resister and two grounded capacitors. The natural frequency and quality factor are electronically tuned. The impedance at input current node exhibits low and the impedance at output current node exhibits high which is convenient to cascade in the current-mode circuit without the use of any current buffer. However, this filter can provide only three filter responses (LP, HP and BP). Also, it requires the multiple output terminal active devices. The three-input single-output current-mode universal filter with five filter responses was proposed in [25]. It consists of single VDCC, a single grounded resister and two grounded capacitors. The natural frequency and quality factor are electronically tuned. The impedance at the output current node exhibits high which is convenient to cascade in the current-mode circuit without the use of any current buffer. However, this filter requires the multiple output terminals of VDCC.

The three-input single-output current-mode biquad filter emphasizing on the use of the VDCC is present in this paper. The proposed filter consists of single VDCC, one resistor and two grounded capacitors which are suitable for the chip and off-the-shelf implementation. The natural frequency and quality factor can be electronically adjusted. The simulation results of the proposed filter agree well with the theoretical expectation.

\section{THE PRINCIPLE OF OPERATION}

\subsection{Voltage differencing current conveyor (VDCC)}

In this design, the active building block $(\mathrm{ABB})$ called the voltage differencing current conveyor (VDCC) is used as the main active device. The international construction of CMOS VDCC was proposed by Firat et al [10] in 2014. It is five port device namely P, N, Z, X and W port. The high impedance voltage input ports are $\mathrm{P}$ and $\mathrm{N}$. The high impedance current output ports are $\mathrm{Z}$ and $\mathrm{W}$ port. The low impedance voltage output port is $\mathrm{X}$ port. In the original version of $\mathrm{VDCC}$, the output current at $\mathrm{W}$ port provides the output current both positive and negative direction called $\mathrm{Wn}$ and $\mathrm{Wp}$ ports. For this purpose, a single $\mathrm{W}$ port is required. This can reduce the current tracking error at $\mathrm{W}$ port and can reduce the number of transistor in the VDCC. The electrical symbol and the equivalent circuit of the VDCC are shown in Figure 1 (a) and (b). The ideal electrical properties of the VDCC are shown in equation (1).

$$
\left(\begin{array}{l}
I_{N} \\
I_{P} \\
I_{Z} \\
V_{X} \\
I_{W}
\end{array}\right)=\left(\begin{array}{cccc}
0 & 0 & 0 & 0 \\
0 & 0 & 0 & 0 \\
g_{m} & -g_{m} & 0 & 0 \\
0 & 0 & 1 & 0 \\
0 & 0 & 0 & 1
\end{array}\right)\left(\begin{array}{l}
V_{P} \\
V_{N} \\
V_{Z} \\
I_{X}
\end{array}\right)
$$

where $g_{m}$ is the transconductance gain. The VDCC can be constructed from commercially available ICs as shown in Figure 1(c). It consists of LM13700 [26] and AD844 [27]. This construction contains only the single $\mathrm{w}$ terminal. The $\mathrm{g}_{\mathrm{m}}$ for this construction is given as

$$
g_{m}=\frac{I_{B}}{2 V_{T}}
$$

where $I_{B}$ is DC bias current and $V_{T}$ is thermal voltage. 


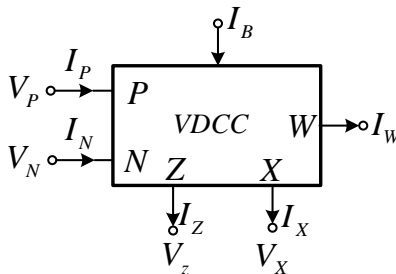

(a)

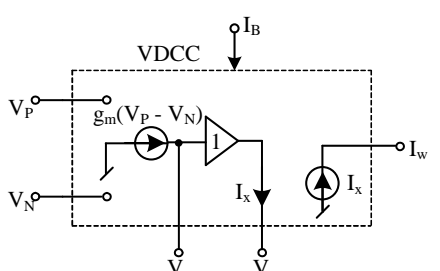

(b)

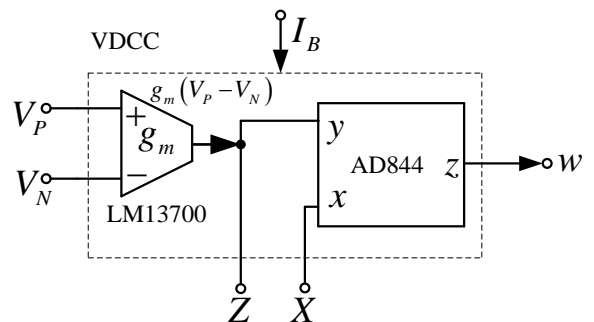

(c)

Figure 1. VDCC (a) Circuit symbol of VDCC (b) Equivalent circuit (c) VDCC (without the dual W terminal) constructed from commercially available ICs

\subsection{Proposed filter}

The proposed filter is shown in Figure 2. It consists of single VDCC, one resistor and two grounded capacitors. There are three input currents, $i_{i 1}, i_{i 2}$ and $i_{i 3}$. The single output current is $i_{\text {out }}$ which exhibits high impedance at the current output node. It is found that the proposed filter doesn't require VDCC with multiple W terminals unlike the current-mode filter using VDCC as the active element presented in [24]-[25]. This makes the VDCC used in the proposed filter is more convenient to construct by using commercially available ICs. Routine analysis of the proposed filter using equation (1) yields the following output current equation

$$
i_{\text {out }}=\frac{i_{i 1} s \frac{1}{C_{1} R}-i_{i 2}\left(s \frac{1}{C_{2} R}+\frac{g_{m}}{C_{1} C_{2} R}\right)+i_{i 3}\left(s^{2}+s \frac{1}{C_{2} R}+\frac{g_{m}}{C_{1} C_{2} R}\right)}{s^{2}+s \frac{1}{C_{2} R}+\frac{g_{m}}{C_{1} C_{2} R}}
$$

From equation (3), the natural frequency and qualigy factor are given as

$$
\omega_{0}=\sqrt{\frac{g_{m}}{C_{1} C_{2} R}} \text { and } Q=\sqrt{\frac{C_{2} g_{m} R}{C_{1}}}
$$

It is evident from equation (4) that the natural frequency and quality factor can be electronically tuned via $\mathrm{g}_{\mathrm{m}}$.

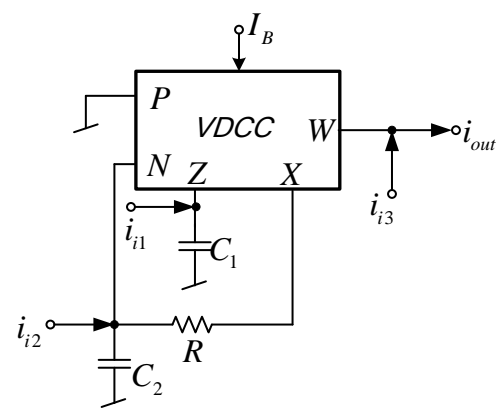

Figure 2. Proposed filter

From equation (3), the derivation of five filter responses can be done as follows:

- The non-inverting band-pass filter response can be obtained by applying the input current to $i_{i 1}$ while $i_{i 2}$ and $i_{i 3}$ being zero (no the input current applying).

- The non-inverting low-pass filter response can be obtained by applying the input current to $i_{i 1}$ and $i_{i 2}$ while $i_{i 3}$ being zero and $C_{1}=C_{2}$.

- The non-inverting high-pass filter response can be obtained by applying the input current to $i_{i 2}$ and $i_{i 3}$ 
while $i_{i l}$ being zero.

- The non-inverting band-reject filter response can be obtained by applying the input current to $i_{i 3}$ and applying the inverting input current to $i_{i 1}$ while $i_{i 2}$ being zero.

- The non-inverting all-pass filter response can be obtained by applying the input current to $i_{i 3}$ and applying the double inverting input current to $i_{i 1}$ while $i_{i 2}$ being zero.

\section{NON-IDEAL ANALYSIS}

In practically, the influent of current/voltage tracking errors and parasitic elements in the VDCC will affect the performances of the proposed filter. For the non-ideal case, the relationship of the terminal voltages and currents of the VDCC can be rewritten as:

$$
\left(\begin{array}{l}
I_{N} \\
I_{P} \\
I_{Z} \\
V_{X} \\
I_{W}
\end{array}\right)=\left(\begin{array}{cccc}
0 & 0 & 0 & 0 \\
0 & 0 & 0 & 0 \\
g_{m} & -g_{m} & 0 & 0 \\
0 & 0 & \beta & 0 \\
0 & 0 & 0 & \alpha
\end{array}\right)\left(\begin{array}{c}
V_{P} \\
V_{N} \\
V_{Z} \\
I_{X}
\end{array}\right)
$$

where $\beta$ is the voltage tracking error from $X$ to $Z$ terminal and $\alpha$ is the current tracking error from $X$ to $W$ terminal. The various ports of VDCC are characterized by parasitic resistances $\left(R_{P}, R_{N}, R_{Z}, R_{X}\right.$ and $\left.R_{W}\right)$ and parasitic capacitances $\left(C_{P}, C_{N}, C_{Z}\right.$, and $\left.C_{W}\right)$ as shown in Figure 3(a). Taking parasitic elements in VDCC into account, the proposed circuit in Figure 2 is re-drawn as shown in Figure 3(b). Taking the non-ideal parameters into account, the output current of the circuit in Figure 2 is obtained as

$$
i_{\text {out }}=\left(\frac{1}{Y_{3} R_{L}+1}\right)\left[\frac{i_{i 1} \alpha \beta Y_{2}-i_{i 2}\left(\alpha Y_{1}+\alpha \beta g_{m}\right)+i_{i 3}\left(Y_{1} Y_{2} R^{\prime}+Y_{1}+\beta g_{m}\right)}{Y_{1} Y_{2} R^{\prime}+Y_{1}+\beta g_{m}}\right]
$$

where $Y_{1}=s\left(C_{l}+C_{z}\right)+G_{z}, Y_{2}=s\left(C_{l}+C_{N}\right)+G_{N}, Y_{3}=s C_{W}+G_{W}, R^{\prime}=R+R_{x}$, and $\mathrm{R}_{\mathrm{L}}$ is the load resistance. If $R_{L}$ is low and the operational frequency $\mathrm{f}_{\mathrm{op}}<<1 / \mathrm{C}_{\mathrm{W}} \mathrm{R}_{\mathrm{W}}$, the output current in equation (6) becomes

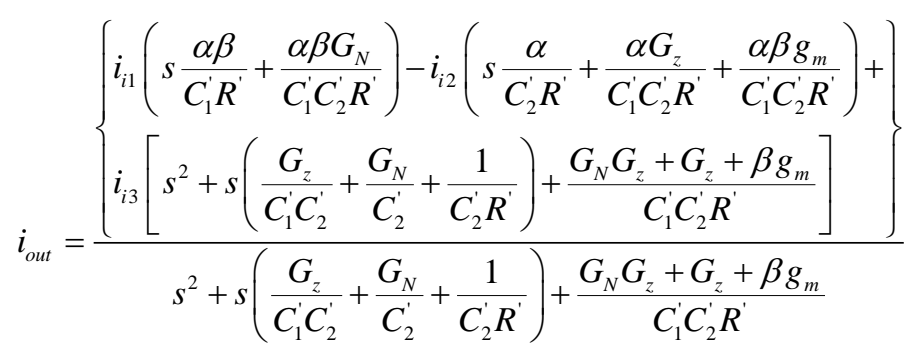

From equation (7), the natural frequency is given as

$$
\omega_{0}=\sqrt{\frac{G_{N} G_{z}+G_{z}+\beta g_{m}}{\left(C_{1}+C_{z}\right)\left(C_{2}+C_{N}\right)\left(R+R_{X}\right)}}
$$

Subsequently, the quality factor is given as

$$
Q=\left[\frac{\left(C_{1}+C_{z}\right)}{G_{z}\left(R+R_{X}\right)+\left(C_{1}+C_{z}\right)\left(R+R_{X}\right) G_{N}+\left(C_{1}+C_{z}\right)} \times \sqrt{\frac{\left(C_{2}+C_{N}\right)\left(R+R_{X}\right)\left(G_{N} G_{z}+G_{z}+\beta g_{m}\right)}{\left(C_{1}+C_{z}\right)}}\right]
$$




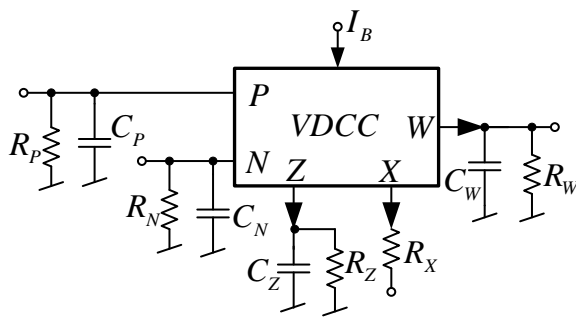

(a)

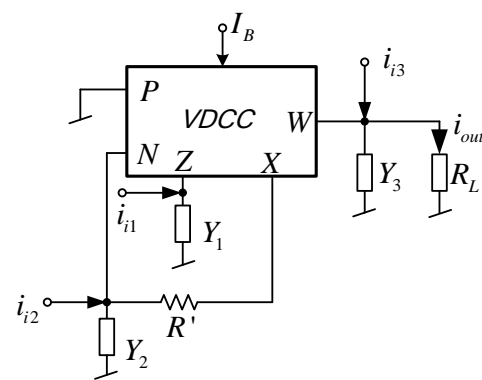

(b)

Figure 3. (a) Parasitic elements appeared in VDCC terminals (b) The proposed filter with parasitic elements

\section{SIMULATION RESULTS}

The simulation was done by using the LM13700 and AD844 PSPICE macro model parameters to confirm the functionality of the proposed filter. The internal construction of the VDCC was constructed as shown in Figure 2(c). The proposed circuit was supplied with symmetrical \pm 5 VDC, the DC bias current was set as $I_{B}=52 \mu \mathrm{A}$ and the passive component values were chosen as $R=1 \mathrm{k} \Omega$ and $C_{l}=C_{2}=1 \mathrm{nF}$. With mentioned active and passive component values, the theoretical natural frequency and quality factor from equation (4) are respectively obtained as $f_{0}=159.23 \mathrm{kHz}, Q=1$.

The simulated gain for LP, HP, BP and BR versus frequency response of the proposed filter with mentioned active and passive component values is shown in Figure 4. The simulated natural frequency is $154.88 \mathrm{kHz}$. The deviation of theoretical and simulated natural frequency is $2.73 \%$. This deviation suffers from the voltage/current tracking error and parasitic elements as analyzed in equation (8). The simulated gain and phase of AP versus frequency response is shown in Figure 5. It is found that phase response changes from $0^{\circ}$ to $-360^{\circ}$ while the magnitude frequency response is constant for all frequencies. The results in Figure 4 and 5 confirm that the proposed circuit can provide five filter responses with the same circuit topology.

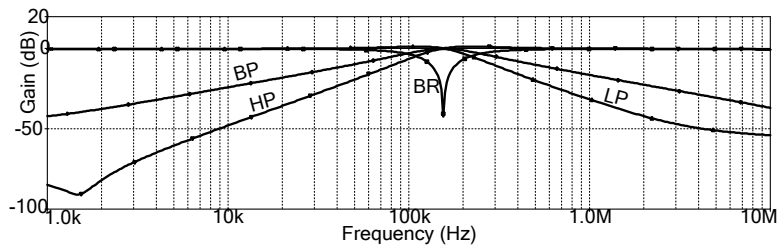

Figure 4. Simulated gain response of the proposed filer

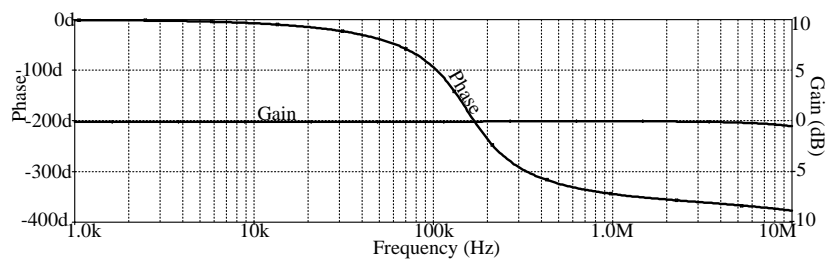

Figure 5. Simulated phase and gain response of all-pass function

The simulated input and output waveform in the time domain for the BP functions is illustrated in Figure 6 when three frequencies, $10 \mathrm{kHz}, 154.88 \mathrm{kHz}$ and $1 \mathrm{MHz}$ with $20 \mu \mathrm{A}_{\mathrm{p}-\mathrm{p}}$ were applied as input current. It is found that the magnitude of output current has the highest amplitude at $\mathrm{f}=154.88 \mathrm{kHz}$. The tuning of phase response for the AP function was tested as shown in Figure 7 by applying the input current as the sinusoidal signal with $20 \mu \mathrm{A}_{\mathrm{p}-\mathrm{p}}$ and $\mathrm{f}=154.88 \mathrm{kHz}$ while the DC bias current was adjusted for three values, 52 $\mu \mathrm{A}, 90 \mu \mathrm{A}$ and $208 \mu \mathrm{A}$. It is found that the phase response of AP function can be controllable with constant 
amplitude. THD analysis of the proposed current-mode universal filter given in Figure 8 was performed at $154.88 \mathrm{kHz}$ for various sinusoidal peak input.

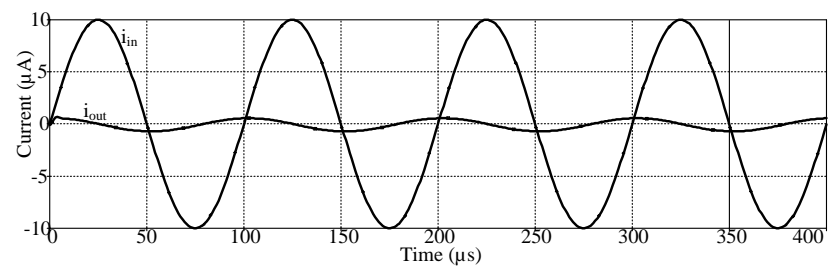

(a)

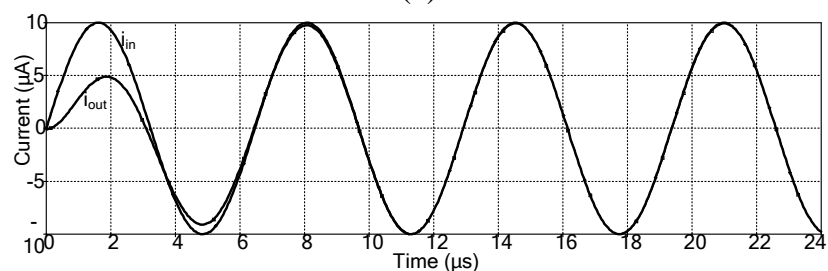

(b)

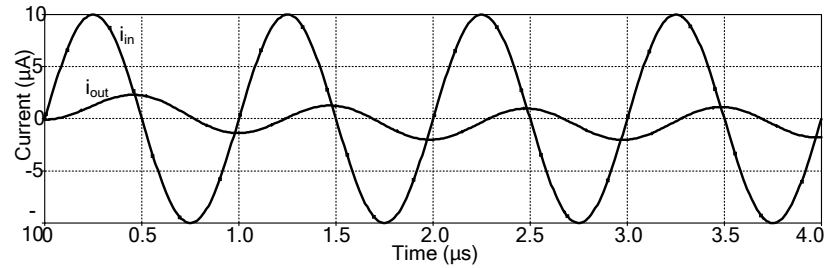

(c)

Figure 6. The simulated waveforms of the BP filter at (a) $10 \mathrm{kHz}$ (b) $154.88 \mathrm{kHz}$ and (c) $1 \mathrm{MHz}$

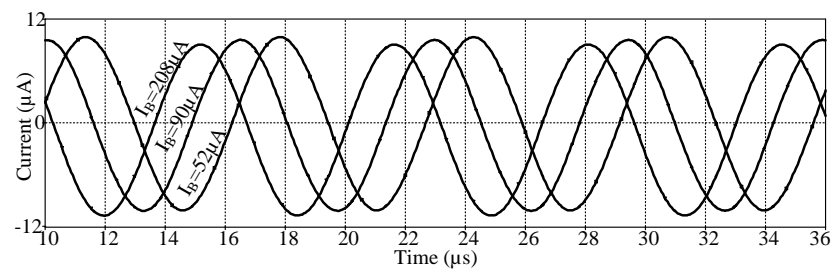

Figure 7. The measured output waveforms of the all-pass filter for different $I_{B}$ values

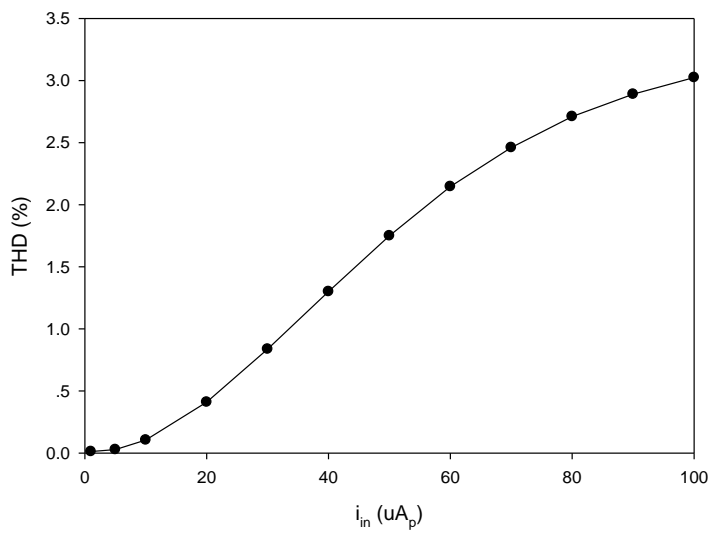

Figure 8. THD variation with respect to applied sinusoidal input current 


\section{EXPERIMENTAL RESULTS}

The proposed filter in Figure 2 was experimentally tested using VDCC construced from the LM13700 and AD844 commercially available ICs as inlustrated in Figure 1(c). The input currents were converted from the input voltage signal by using the AD844 IC and one resistor as shown in Figure 9. The $1 \mathrm{k} \Omega$ resistor was used as the output load to convert the output current to be the output voltage. The LM13700 and AD844 were biased with a DC supply voltage of $\pm 5 \mathrm{~V}$ and bias current of $\mathrm{I}_{\mathrm{B}}=52 \mu \mathrm{A}$. All capacitances and resistance were given by $1 \mathrm{nF}$ and $1 \mathrm{k} \Omega$, respectively. The amplitude of input voltage for this test was 50 $\mathrm{mV}_{\mathrm{p}-\mathrm{p}}$. The experimentally observed frequency response of the LP, HP, BP and BR filter is shown in Figure 10. The measured $\mathrm{f}_{0}$ was obtained around $160 \mathrm{kHz}$. The measurement of the input voltage and output voltage dropped at the output resistance load for the BP response is shown in Figure 11. The experimentally measured gain and phase response of the AP filter is illustrated in Figure 12. It is found that experimental results are in well accordance to the theoretical analyses.

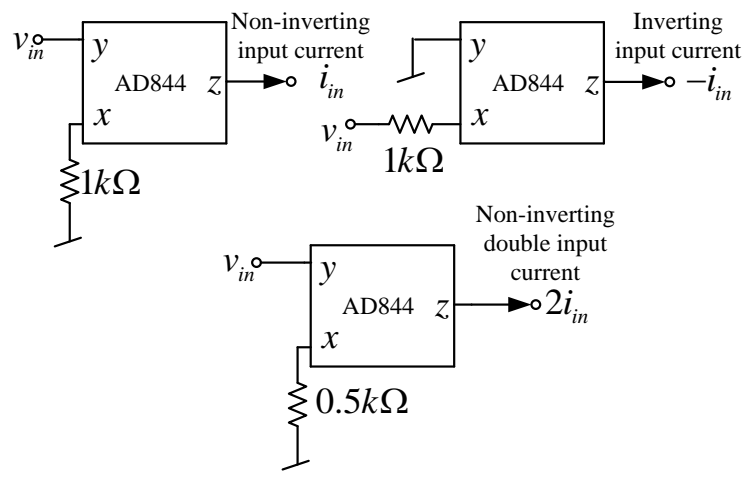

Figure 9. Implementation of input currents using AD844

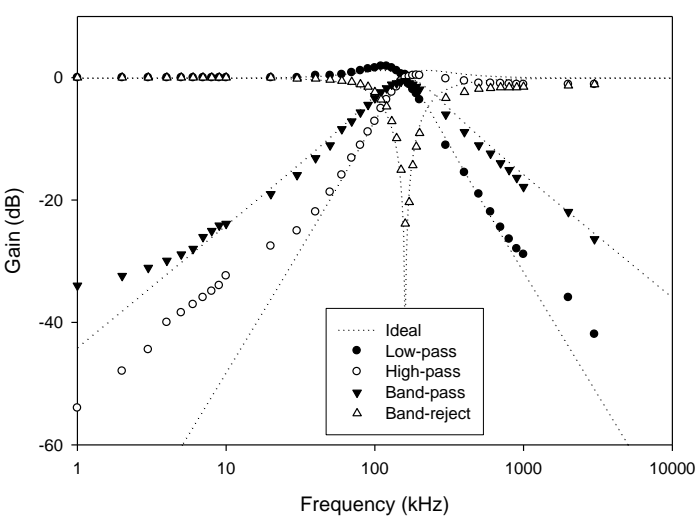

Figure 10. Experimental gain response of the proposed filer

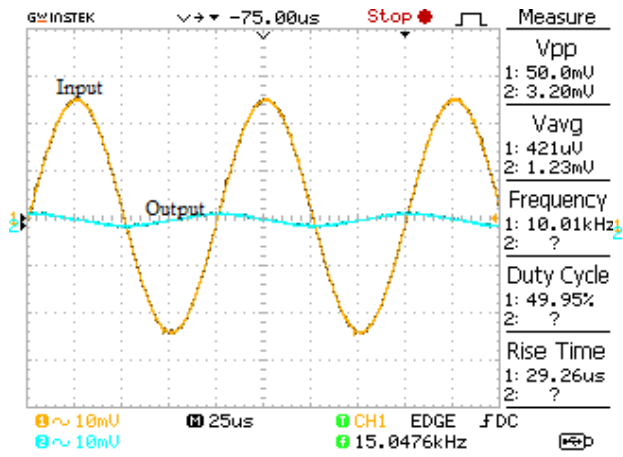

(a)

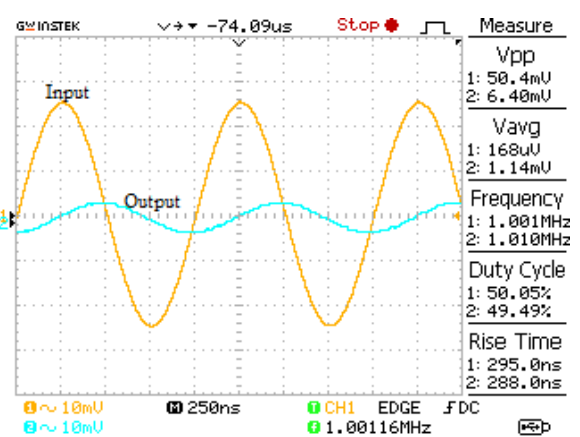

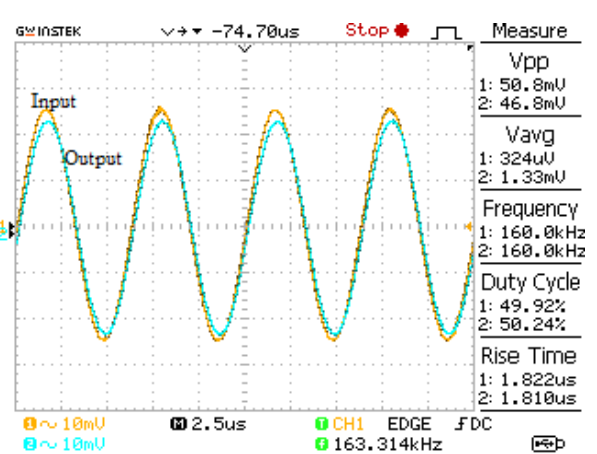

(b)

(c)

Figure 11. The input and output waveforms of BP filter at (a) $10 \mathrm{kHz}$ (b) $160 \mathrm{kHz}$ and (c) $1 \mathrm{MHz}$. 


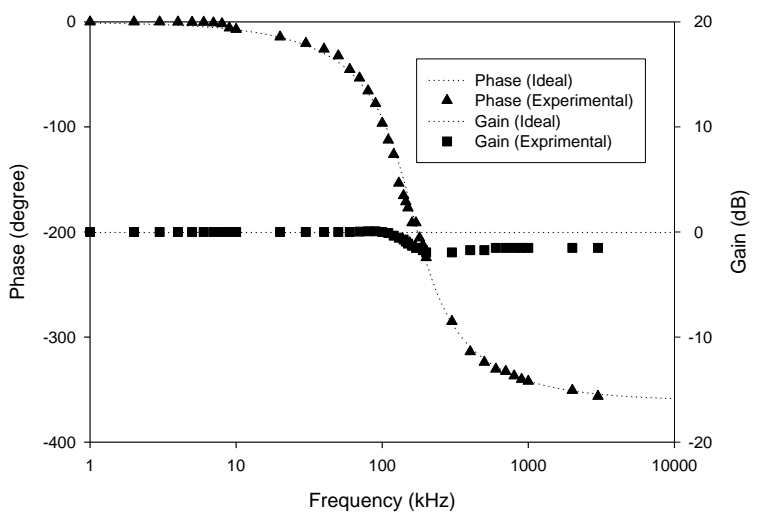

Figure 12. Experimental phase and gain response of the all-pass function

\section{CONCLUSION}

In this contribution, the three-input single-output current-mode filter is presented. The proposed filter uses only single VDCC as active element. The natural frequency and quality factor can be tuned electronically by changing the bias current of VDCC. Using only VDCC constructed from commercially available ICs, the proposed filter is suitable for off-the-shelf implementation. The workability of the proposed filter is demonstrated by simulation and experimental results.

\section{REFERENCES}

[1] R. Tiwari and G. R. Mishra, "A novice approach of designing CMOS based switchable filters for ASP applications," International Journal of Electrical and Computer Engineering (IJECE), vol. 5, pp. 1354-1362, 2015.

[2] S. Siripongdee and W. Jaikla, "Universal filter using single commercially available IC: LT1228," 3rd International Conference on Mechatronics and Mechanical Engineering, Shanghai; China, pp. 1-4, 2016.

[3] C. Toumazou, et al., "Analogue IC design: The Current-mode Approach," Peter Peregrinus, London, U.K, 1990.

[4] R. Arslanalp, et al., "Two lossy integrator loop based current-mode electronically tunable universal filter employing only grounded capacitors," Microelectronics Journal, vol. 59, pp. 1-9, 2017.

[5] D. Biolek, et al., "Active elements for analog signal processing, classification, review and new proposals," Radioengineering, vol/issue: 17(4), pp. 15-32, 2008.

[6] G. Komanaplli, et al., "New realization of quadrature oscillator using OTRA," International Journal of Electrical and Computer Engineering (IJECE), vol. 7, pp. 1815-1823, 2017.

[7] J. Sharma and S. Chakraborty, "MOCCCDTA-based current mode tunable universal biquad filter for bluetooth applications," International Journal of Electrical and Computer Engineering, vol. 6, pp. 1499-1505, 2016.

[8] W. Jaikla, et al., "Electronically tunable current-mode biquad filter employing CCCDTAs and grounded capacitors with low input and high output impedance," AEU - International Journal of Electronics and Communications, vol/issue: 67(12), pp. 1005-1009, 2013.

[9] W. Jaikla, et al., "High input impedance voltage-mode biquad filter using VD-DIBAs," Radioengineering, vol/issue: 23(3), pp. 914-921, 2014.

[10] F. Kacar, et al., "Positive/negative lossy/lossless grounded inductance simulators employing single VDCC and only two passive elements," AEU-International Journal of Electronics and Communications, vol/issue: 68(1), pp. 73-78, 2014.

[11] R. Sotner, et al., "Novel first-order all-pass filter applications of z-copy voltage differencing current conveyor," Indian Journal of Pure and Applied Physics, vol/issue: 53(8), pp. 537-545, 2015.

[12] D. Prasad, et al., "Novel VDCC based low-pass and high-pass ladder filters," 12th IEEE International Conference Electronics, Energy, Environment, Communication, Computer, Control: (E3-C3), 2016.

[13] A. Kartci, et al., "Application possibilities of VDCC in general floating element simulator circuit," European Conference on Circuit Theory and Design, 2015.

[14] A. Kartci, et al., "Electronically tunable VDCC-based floating capacitance multiplier," 23rd Signal Processing and Communications Applications Conference, pp. 2474-2477, 2015.

[15] A. Kartci, et al., "Floating capacitance multiplier simulator for grounded RC colpitts oscillator design," International Conference on Applied Electronics, pp. 93-96, 2015.

[16] D. Prasad and J. Ahmad, "New electronically-controllable lossless synthetic floating inductance circuit using single VDCC," Circuits and Systems, vol. 5, pp. 13-17, 2014.

[17] R. Sotner, et al., "Design of Z-copy controlled-gain voltage differencing current conveyor based adjustable functional generator," Microelectronics Journal, vol/issue: 46(2), pp. 143-152, 2015. 
[18] D. Prasad, et al., "New single VDCC-based explicit current-mode SRCO employing all grounded passive components," Electronics, vol/issue: 18(2), pp. 81-88, 2014.

[19] R. Sotner, et al., "Simple CMOS voltage differencing current conveyor-based electronically tunable quadrature oscillator," Electronics Letters, vol/issue: 52(12), pp. 1016-1018, 2016.

[20] M. Srivastava and D. Prasad, "VDCC based dual-mode quadrature sinusoidal oscillator with outputs at appropriate impedance levels," Advances in Electrical and Electronic Engineering, vol/issue: 14(2), pp. 168-177, 2016.

[21] R. Sotner, et al., "Voltage differencing current conveyor based linearly controllable quadrature oscillators," International Conference on Applied Electronics, pp. 237-240, 2016.

[22] F. Kaçar, et al., "Design and experiment of VDCC-based voltage mode universal filter," Indian Journal of Pure and Applied Physics, vol/issue: 53(5), pp. 341-349, 2015.

[23] M. Sagbas, et al., "Electronically tunable universal biquad using a single active component," 38th International Conference on Telecommunications and Signal Processing, pp. 698-702, 2015.

[24] J. Jerabek, et al., "Reconnection-less electronically reconfigurable filter with adjustable gain using voltage differencing current conveyor," Elektronika ir Elektrotechnika, vol/issue: 22(6), pp. 39-45, 2016.

[25] P. Lamun, et al., "Single VDCC-based current-mode universal biquadratic filter," 7th International Conference on Information Technology and Electrical Engineering: Envisioning the Trend of Computer, Information and Engineering, pp. 122-125, 2016.

[26] http://www.ti.com/lit/ds/symlink/lm13700.pdf

[27] http://www.analog.com/media/en/technical-documentation/data-sheets/AD844.pdf 